\title{
Síndrome Metabólica em Portugal: Prevalência e Fatores Associados
}

\author{
Metabolic Syndrome in Portugal: Prevalence and \\ Associated Factors
}

\author{
Ricardo ALVES ${ }^{1}$, Ana João SANTOS², Irina KISLAYA², Baltazar NUNES², Ana Cristina FREIRE1 \\ Acta Med Port 2022 Sep;35(9):633-643 - https://doi.org/10.20344/amp.15051
}

\section{RESUMO}

Introdução: A síndrome metabólica consiste num conjunto de fatores que, quando associados, conferem maior risco de desenvolver doenças cardiovasculares e diabetes tipo 2, constituindo um importante problema de saúde pública. O objetivo deste estudo foi estimar a prevalência desta síndrome na população portuguesa, e avaliar possíveis associações com determinantes demográficos e socioeconómicos.

Material e Métodos: Com base no primeiro Inquérito Nacional de Saúde com Exame Físico de 2015, realizou-se um estudo epidemiológico transversal numa amostra representativa da população portuguesa $(n=4797)$ entre os 25 e 74 anos. A prevalência foi estimada na população total e em cada sexo, estratificada por grupo etário, região de saúde, tipologia de área urbana, estado civil, escolaridade, situação profissional e risco de pobreza. A magnitude das associações foi medida pelas razões de prevalências ajustadas.

Resultados: A prevalência estimada foi de 33,4\% (IC 95\%, 31,7 - 35,1) na população portuguesa [35,6\% nos homens (IC 95\%, 31,9 - 39,2) e 31,3\% nas mulheres (IC 95\%, 28,5 - 34,2)]. Em ambos os sexos, a maior prevalência estava significativamente associada ao aumento da idade, a indivíduos viúvos/casados/unidos de facto e com menor escolaridade. Não se verificou associação com sexo, região de saúde, tipologia de área urbana, situação profissional ou risco de pobreza.

Conclusão: A síndrome metabólica estava independentemente associada a grupos específicos. Este conhecimento reforça a importância de uma avaliação holística dos determinantes de saúde associados à síndrome metabólica.

Palavras-chave: Portugal; Prevalência; Síndrome Metabólica/epidemiologia

\section{ABSTRACT}

Introduction: The metabolic syndrome consists of a set of factors that, when associated, are associated with a higher risk of developing cardiovascular diseases and type 2 diabetes, and is thus an important public health problem. The objective of this study was to estimate the prevalence of this syndrome in the Portuguese population, and to evaluate possible associations with demographic and socioeconomic determinants.

Material and Methods: Based on the $1^{\text {st }}$ National Health Survey with Physical Examination of 2015, a cross-sectional epidemiological study was conducted on a representative sample of the Portuguese population $(n=4797)$ aged between 25 and 74 years old. The prevalence was estimated for the total population and each gender, stratified by age group, health region, type of urban area, marital status, education, professional status, and risk of poverty. The magnitude of the associations was measured with adjusted prevalence ratios. Results: In the Portuguese population the estimated prevalence was $33.4 \%$ [95\% Cl, $31.7-35.1][35.6 \%$ in men $(95 \% \mathrm{Cl}, 31.9-39.2)$ and $31.3 \%$ in women $(95 \% \mathrm{Cl}, 28.5-34.2)]$. In both genders, the highest prevalence was significantly associated with increasing age, widowed/married/de facto partners and those with lower levels of education. There was no association with gender, health region, type of urban area, professional status or risk of poverty.

Conclusion: Metabolic syndrome was independently associated with specific groups. This knowledge reinforces the importance of a holistic assessment of the health determinants associated with the metabolic syndrome.

Keywords: Metabolic Syndrome/epidemiology; Portugal; Prevalence

\section{INTRODUÇÃO}

A síndrome metabólica (SM) tem elevada prevalência a nível global, constituindo um problema atual de saúde pública pela morbilidade e mortalidade associadas. ${ }^{1}$ Esta síndrome duplica o risco potencial de desenvolvimento de doenças cardiovasculares nos cinco a 10 anos seguintes e está associado a um aumento de cinco vezes no risco de desenvolver diabetes tipo 2 ao longo da vida quando comparados com indivíduos sem SM. ${ }^{2}$

Diferentes organizações propuseram critérios distintos para a definição da SM. Contudo, um consenso entre várias sociedades internacionais foi estabelecido em 2009, criando uma definição harmonizada. Segundo esta definição, a
SM consiste na presença em simultâneo de três ou mais das seguintes componentes: hiperglicemia, hipertensão arterial (HTA), hipertrigliceridemia, níveis baixos de colesterol de lipoproteína de alta densidade (c-HDL) e perímetro da cintura aumentado. ${ }^{2}$

A SM é comum e tem uma prevalência crescente em todo o mundo, que se relaciona em grande parte com o aumento da obesidade e com os estilos de vida sedentários. ${ }^{2-4}$ Estima-se que a sua prevalência a nível global seja de $20 \%-25 \% .^{5-7}$ O estudo Portuguese Metabolic Syndrome (PORMETS), realizado em 2007/2009 na população com 18 ou mais anos de idade, estimou uma prevalência da

1. Unidade de Saúde Pública. Agrupamento de Centros de Saúde Almada-Seixal. Almada. Portugal.

2. Departamento de Epidemiologia. Instituto Nacional de Saúde Doutor Ricardo Jorge. Lisboa. Portugal.

$\triangle$ Autor correspondente: Ricardo Alves. ricardoalves.medicina@gmail.com

Recebido/Received: 06/10/2020 - Aceite/Accepted: 16/04/2021 - Publicado Online/Published Online: 06/10/2021 - Publicado/Published: 01/09/2022 Copyright $\odot$ Ordem dos Médicos 2022 
SM de $43,1 \%$ (39,8\% nos homens e $45,7 \%$ nas mulheres) em Portugal Continental, utilizando a definição harmonizada. ${ }^{8}$ O Estudo Epidemiológico de Prevalência da Síndrome Metabólica na População Portuguesa (VALSIM), realizado em 2006/2007, analisou os utentes com 18 ou mais anos de idade frequentadores dos cuidados de saúde primários (CSP) em Portugal Continental e Ilhas e, aplicando critérios semelhantes à definição harmonizada, estimou uma prevalência da SM de $27,5 \%$ (26,0\% nos homens e $28,7 \%$ nas mulheres). ${ }^{9}$ Por outro lado, em 2015 , segundo o $1^{\circ} \mathrm{In}$ quérito Nacional de Saúde com Exame Físico (INSEF) a prevalência de obesidade ou excesso de peso em Portugal correspondia a cerca de dois terços da população adulta $(67,6 \%){ }^{10}$

Uma recente meta-análise mostrou um aumento da prevalência de SM em indivíduos com vulnerabilidade socioeconómica [OR 1,15 (IC 95\%, 1,12 - 1,18)]. . ${ }^{11}$ Os determinantes demográficos e socioeconómicos foram abordados por vários estudos que mostraram consistentemente o aumento da prevalência da SM nos grupos etários com mais idade ${ }^{7-9}$ menor escolaridade ${ }^{12-14}$ e situação laboral e financeira desfavorecida. ${ }^{15}$

Dado que as últimas estimativas da prevalência da SM na população portuguesa reportam a $2007 / 2009,{ }^{8}$ o objetivo do presente estudo foi determinar a prevalência da SM e seus fatores associados na população residente em Portugal em 2015, com idades entre os 25 e os 74 anos.

\section{MATERIAL E MÉTODOS Fonte dos dados}

Este estudo epidemiológico observacional transversal descritivo com componente analítica teve como base o INSEF de 2015. ${ }^{16}$ O INSEF foi desenvolvido, entre 2013 e 2016, pelo Instituto Nacional de Saúde Doutor Ricardo Jorge (INSA) em colaboração com as cinco administrações regionais de saúde do Continente, as secretarias regionais de saúde e dos assuntos sociais das regiões autónomas dos Açores e da Madeira e o Instituto Norueguês de Saúde Pública. Todos os procedimentos foram realizados segundo as recomendações do Inquérito Europeu de Saúde com Exame Físico (EHES). ${ }^{17}$ O INSEF incluiu três componentes: exame físico, colheita de sangue para análises clínicas e entrevista com um questionário geral de saúde. O trabaIho de campo decorreu entre fevereiro e dezembro de 2015 e foi realizado por equipas formadas e treinadas especificamente para o INSEF, num total de 117 profissionais de saúde. O exame físico (medição da tensão arterial, altura, peso e perímetros da cintura e anca) foi realizado por enfermeiros e seguiu todos os procedimentos e recomendações do EHES. A tensão arterial foi medida, após repouso de cinco minutos na posição sentada, por três vezes no braço direito com um minuto de intervalo entre medições. As coIheitas de sangue para análises clínicas [perfil lipídico, hemoglobina glicada A1c (HbA1c) e hemograma] foram realizadas por um técnico de análises clínicas ou um enfermeiro e decorreram durante todo o dia, sem jejum obrigatório. $O$ questionário, baseado em instrumentos padronizados e amplamente testados, foi aplicado por Computer-Assisted Personal Interview e incluiu 23 secções (relativas a caraterização sociodemográfica e socioeconómica, estado de saúde, determinantes de saúde e cuidados de saúde).

\section{População em estudo}

A população alvo do INSEF foi constituída por indivíduos entre os 25 e 74 anos, que em 2015 residiam em Portugal há pelo menos 12 meses antes da data da entrevista, não-institucionalizados, e que falavam a língua portuguesa. Os participantes do INSEF foram selecionados em duas etapas, através de amostragem probabilística estratificada por região (Norte, Centro, Lisboa e Vale do Tejo, Alentejo, Algarve, Madeira e Açores) e tipologia de área urbana (rural e urbana). Numa primeira etapa, em cada região foram selecionadas aleatoriamente sete unidades primárias de amostragem (PSU), geralmente definidas por áreas geográficas correspondentes à área de influência dos antigos centros de saúde do Serviço Nacional de Saúde, com probabilidade de seleção proporcional à dimensão da população residente com idade elegível. Na segunda etapa, em cada unidade primária de amostragem foram selecionados indivíduos por amostragem aleatória simples, que constituem as unidades secundárias de amostragem, a partir das listas de utentes do Serviço Nacional de Saúde. A dimensão da amostra foi estabelecida de forma a ser possível estimar uma prevalência esperada de $50 \%$, com uma precisão absoluta de $5 \%$ para um intervalo de confiança a $95 \%$, em cada região de saúde do Continente ou Região Autónoma, considerando um efeito do desenho da amostra de 1,5. O cálculo da dimensão da amostra mínima necessária resultou em 600 indivíduos a nível regional e 4200 a nível nacional, sendo que a amostra final obtida no INSEF correspondeu a 4911 indivíduos. ${ }^{18}$

Para o presente estudo foram excluídos os indivíduos cujas respostas omissas não permitiam determinar a presença ou ausência de SM $(n=114)$, correspondendo a uma amostra final de 4797 indivíduos.

\section{Variáveis em estudo}

As caraterísticas demográficas e socioeconómicas incluíram o sexo, grupo etário (25 - 44; 45 - 54; 55 - 64; 65 - 74), região de residência (Norte, Centro, Lisboa e Vale do Tejo, Alentejo, Algarve, Região Autónoma da Madeira e Região Autónoma dos Açores), tipologia de área urbana (rural e urbana), estado civil (solteiro; casado ou em união de facto; divorciado; viúvo), nível de escolaridade (analfabeto $/ 1^{\circ}$ ciclo do ensino básico; $2^{\circ}$ ciclo $/ 3^{\circ}$ ciclo do ensino básico; secundário; ensino superior), condição perante o trabalho e risco de pobreza. Para a variável tipologia de área urbana, as áreas de influência das PSU foram analisadas a partir de informação dos Censos (2011) e da tipologia de área urbana (TIPAU), tendo uma PSU sido classificada como urbana quando o peso da população residente com idade elegível nas freguesias com classificação TIPAU de área predominantemente urbana era superior a $50 \%$ da população total da PSU; caso contrário, a PSU seria 
classificada como rural. A condição perante o trabalho foi definida em três categorias - trabalho remunerado, desempregado e sem atividade profissional remunerada (inclui reformados, domésticas e estudantes). O risco de pobreza foi aferido com a questão: "Se surgisse uma despesa inesperada de cerca de 434 euros, conseguiria o seu agregado familiar pagá-la de imediato sem recorrer a empréstimos?". Este item utiliza uma percentagem do ordenado mínimo, e faz parte dos itens de privação material na população total do European Union Statistics on Income and Living Conditions (EU-SILC) 2014 - 2015 para avaliar o risco do limiar da pobreza. ${ }^{19}$

As variáveis do exame físico incluíram o perímetro da cintura $(\mathrm{cm})$ e tensão arterial sistólica e diastólica $(\mathrm{mmHg})$.

As variáveis em estudo obtidas através da colheita de sangue para análises clínicas foram a trigliceridemia (mg/ $\mathrm{dL})$, o c-HDL (mg/dL) e a HbA1c (\%).

Considerou-se SM presente quando um indivíduo tinha simultaneamente três ou mais das cinco componentes seguintes: perímetro da cintura aumentado (homens $\geq 94 \mathrm{~cm}$ e mulheres $\geq 80 \mathrm{~cm}$ ), trigliceridemia $\geq 175 \mathrm{mg} / \mathrm{dL}$, c-HDL diminuído (homens $<40 \mathrm{mg} / \mathrm{dL}$ e mulheres $<50 \mathrm{mg} / \mathrm{dL}$ ), HTA (sistólica $\geq 130 \mathrm{mmHg}$, diastólica $\geq 85 \mathrm{mmHg}$ ou terapêutica anti-hipertensora) e $\mathrm{HbA} 1 \mathrm{c} \geq 5,7 \%$ ou terapêutica para glicemia elevada. Devido ao jejum não estar garantido, o ponto de corte considerado para trigliceridemia elevada foi de $\geq 175 \mathrm{mg} / \mathrm{dL}$ (em vez de $\geq 150 \mathrm{mg} / \mathrm{dL}$ como indicado na definição harmonizada). Não foi realizada qualquer correção ao valor do c-HDL, pois este não é afetado pelo estado de jejum/não jejum. ${ }^{20}$ Nas variáveis trigliceridemia e c-HDL não se considerou a toma de medicação, uma vez que esta informação era omissa no questionário geral de saúde. A HbA1c substituiu a componente da definição harmonizada 'Glicemia em jejum $\geq 100 \mathrm{mg} / \mathrm{dL}$ ', tendo-se selecionado o ponto de corte de $\geq 5,7 \%$.

\section{Análise estatística}

É consensual a existência de diferenças entre sexos em termos de estado de saúde e comportamentos relacionados com saúde. Estas diferenças podem dever-se a aspetos biológicos ou determinantes comportamentais. ${ }^{21,22}$ Dada a etiologia multifatorial da SM, considerou-se mais adequada a análise das estimativas estratificada por sexo.

A população em estudo foi caraterizada segundo as variáveis demográficas, socioeconómicas, analíticas e do exame físico. Para as variáveis nominais e ordinais foram criadas tabelas de distribuição de frequências com as contagens e as respetivas percentagens. Para as variáveis numéricas foram determinadas medidas de tendência central como a média e mediana, e medidas de dispersão, como o desvio padrão, amplitude, mínimo e máximo.

A prevalência da SM, e respetivos intervalos de confiança a 95\% (IC 95\%), foi estimada para o total da população e separadamente para homens e mulheres, estratificada por grupo etário, região de saúde, tipologia de área urbana, estado civil, escolaridade, condição perante o trabalho e risco de pobreza. A versão de Rao-Scott do teste qui-quadrado ajustada ao desenho amostral foi utilizada para comparar as prevalências estimadas entre as categorias das variáveis de estratificação. ${ }^{23}$

Para identificar fatores associados à SM foram estimadas as razões de prevalência bruta (RP), de acordo com grupo etário, região de saúde, tipologia de área urbana, estado civil, escolaridade, condição perante o trabalho e risco de pobreza, usando a regressão de Poisson. Estimaram-se também as razões de prevalências ajustadas com respetivos IC 95\% [aRP (IC 95\%)]: ajustadas, no total da população, para sexo e grupo etário; ajustadas para grupo etário no sexo; as variáveis 'trabalho' e 'risco de pobreza' foram também ajustadas para o nível de escolaridade. Optou-se pelo modelo de regressão de Poisson devido à expectável elevada prevalência da SM (> 10\%), que utilizando a odds ratio (OR) obtida através de regressão logística poderia levar à sobrestimação das magnitudes de associação. ${ }^{24}$ Foi considerado o nível de significância de $5 \%$.

Todas as estimativas pontuais foram ajustadas utilizando pesos amostrais calibrados para a distribuição da população portuguesa, por sexo e grupo etário, em cada uma das sete regiões de saúde, para a estimativa da população residente em 2014.

A análise estatística foi realizada com recurso ao software R (versão 1.1.463) ${ }^{25}$ e módulo survey (versão 3.36) ( $^{26}$ para análise de amostras complexas.

\section{Questões éticas}

Este estudo utilizou a base de dados do INSEF, que foi criada seguindo um protocolo autorizado pela Comissão Nacional de Proteção de Dados e pelas Comissões de Ética para a Saúde do INSA, Administrações Regionais de Saúde do Norte, Centro, Lisboa e Vale do Tejo, Alentejo e Algarve, Serviço de Saúde da Região Autónoma da Madeira, Hospital da Horta e Centro Hospitalar de Lisboa Ocidental. Todos os participantes no INSEF assinaram uma declaração de consentimento informado referente ao exame físico, colheita de sangue e entrevista de saúde.

Foi obtido o parecer positivo da Comissão de Ética para a Saúde do INSA para a realização do presente estudo.

\section{RESULTADOS \\ Caraterização da amostra em estudo}

A amostra em estudo compreendeu 4797 indivíduos, sendo $52,1 \%$ do sexo feminino (Tabela 1 ). A maioria dos indivíduos da amostra tinha menos de 55 anos (64,6\%), era casada ou unida de facto $(70,1 \%)$, residia em área urbana $(71,5 \%)$, tinha trabalho remunerado $(61,0 \%)$ e conseguia suportar uma despesa inesperada $(60,9 \%)$. Cerca de um terço tinha o $2^{\circ}$ ou $3^{\circ}$ ciclo do ensino básico $(32,7 \%)$ e apenas $17,3 \%$ frequentou ou completou o ensino superior.

A Tabela 2 apresenta os resultados das caraterísticas analíticas, tensão arterial e medições antropométricas da amostra, segundo os critérios relevantes para a definição da SM. No total da amostra, o perímetro da cintura aumentado foi a componente mais prevalente $(64,5 \%)$ e o c-HDL diminuído a menos frequente $(24,2 \%)$. A distribuição das 
Tabela 1 - Distribuição das características demográficas e socioeconómicas da amostra de indivíduos entre 25 e 74 anos de Portugal $(n=4797)$, estratificadas por sexo. (2015)

\begin{tabular}{|c|c|c|c|c|c|c|c|c|}
\hline \multirow{2}{*}{ Variável } & \multirow{2}{*}{ Valores } & \multicolumn{2}{|c|}{ Total } & \multicolumn{2}{|c|}{ Feminino } & \multicolumn{2}{|c|}{ Masculino } & \multirow{2}{*}{$p^{*}$} \\
\hline & & $\mathbf{n}$ & $\%$ & $\mathbf{n}$ & $\%$ & $\mathbf{n}$ & $\%$ & \\
\hline \multirow[t]{2}{*}{ Sexo } & Feminino & 2571 & 53,6 & 2571 & 100,0 & - & - & - \\
\hline & Masculino & 2226 & 46,4 & - & - & 2226 & 100,0 & - \\
\hline \multirow[t]{4}{*}{ Grupo etário } & $25-44$ anos & 1830 & 42,3 & 997 & 38,8 & 833 & 37,4 & $<0,001$ \\
\hline & $45-54$ anos & 1161 & 22,3 & 633 & 24,6 & 528 & 23,7 & 0,002 \\
\hline & $55-64$ anos & 1067 & 19,9 & 562 & 21,9 & 505 & 22,7 & 0,081 \\
\hline & $65-75$ anos & 739 & 15,5 & 379 & 14,7 & 360 & 16,2 & 0,485 \\
\hline \multirow[t]{7}{*}{ Região } & Norte & 772 & 16,1 & 438 & 17,0 & 334 & 15,0 & $<0,001$ \\
\hline & Centro & 695 & 14,5 & 358 & 13,9 & 337 & 15,1 & 0,426 \\
\hline & Lisboa e Vale do Tejo & 614 & 12,8 & 327 & 12,7 & 287 & 12,9 & 0,106 \\
\hline & Alentejo & 660 & 13,8 & 350 & 13,6 & 310 & 13,9 & 0,120 \\
\hline & Algarve & 635 & 13,2 & 324 & 12,6 & 311 & 14,0 & 0,606 \\
\hline & Região Autónoma da Madeira & 688 & 14,3 & 373 & 14,5 & 315 & 14,2 & 0,027 \\
\hline & Região Autónoma dos Açores & 733 & 15,3 & 401 & 15,6 & 332 & 14,9 & $<0,001$ \\
\hline \multirow{2}{*}{$\begin{array}{l}\text { Tipologia área } \\
\text { urbana }\end{array}$} & Rural & 1368 & 28,5 & 735 & 28,6 & 633 & 28,4 & 0,006 \\
\hline & Urbana & 3429 & 71,5 & 1836 & 71,4 & 1593 & 17,6 & $<0,001$ \\
\hline \multirow[t]{4}{*}{ Estado civil } & Solteiro & 800 & 16,7 & 385 & 15,0 & 415 & 18,6 & 0,289 \\
\hline & Casado ou união de facto & 3365 & 70,1 & 1749 & 68,0 & 1616 & 72,6 & 0,022 \\
\hline & Divorciado & 396 & 8,3 & 235 & 9,1 & 161 & 7,2 & $<0,001$ \\
\hline & Viúvo & 236 & 4,9 & 202 & 7,9 & 34 & 1,5 & $<0,001$ \\
\hline \multirow[t]{4}{*}{ Escolaridade } & Analfabeto $/ 1^{\circ}$ ciclo & 1458 & 30,4 & 760 & 29,6 & 698 & 31,4 & 0,104 \\
\hline & $2^{\circ}$ ciclo $/ 3^{\circ}$ ciclo & 1568 & 32,7 & 773 & 30,1 & 795 & 35,7 & 0,578 \\
\hline & Ensino secundário & 939 & 19,6 & 502 & 19,5 & 437 & 19,6 & 0,034 \\
\hline & Ensino superior & 829 & 17,3 & 533 & 20,8 & 296 & 13,3 & $<0,001$ \\
\hline \multirow[t]{3}{*}{ Trabalho } & Trabalho remunerado & 2925 & 61,0 & 1501 & 58,4 & 1424 & 64,0 & 0,154 \\
\hline & Desempregado & 536 & 11,2 & 298 & 11,6 & 238 & 10,7 & 0,010 \\
\hline & Outra sem atividade profissional & 1334 & 27,8 & 770 & 30,0 & 564 & 25,3 & $<0,001$ \\
\hline \multirow[t]{2}{*}{ Risco pobreza } & Não suporta despesa inesperada & 1853 & 39,1 & 1103 & 43,5 & 750 & 34,0 & $<0,001$ \\
\hline & Suporta despesa inesperada & 2886 & 60,9 & 1433 & 56,5 & 1453 & 66,0 & 0,710 \\
\hline
\end{tabular}

* Comparação de proporções entre sexo feminino e masculino

componentes alteradas diferiu entre sexos, verificando-se as maiores diferenças ao nível da hipertrigliceridemia ( $37,2 \%$ nos homens vs $20,9 \%$ nas mulheres) e HTA $(58,2 \%$ nos homens vs $41,4 \%$ nas mulheres).

Entre os indivíduos da amostra com SM, a componente mais prevalente foi o perímetro de cintura aumentado $(95,4 \%)$, seguida da hipertrigliceridemia $(84,5 \%)$, c-HDL diminuído $(62,1 \%)$ e HTA $(61,8 \%)$, enquanto a HbA1c aumentada foi a componente menos frequente $(50,4 \%)$. Mais de metade dos indivíduos cumpria os critérios de SM pela presença de três das cinco componentes $(58,1 \%)$ e 10,4\% apresentava todas as componentes.

\section{Prevalência de síndrome metabólica}

A estimativa de prevalência de SM foi de $33,4 \%$ (IC $95 \%, 31,7$ - 35,1) (Tabela 3) para a população com idade entre 25 e 74 anos. Não se observaram diferenças estatisticamente significativas entre os sexos, sendo a esti- mativa pontual da prevalência nos homens de $35,6 \%$ (IC $95 \%, 31,9-39,2$ ) e nas mulheres de $31,3 \%$ (IC 95\%, 28,5 - 34,2). Observou-se um gradual aumento da prevalência dos grupos etários mais jovens para os mais velhos [14,5\% vs 59,8\%; RP $=4,13(3,51-4,90), p<0,001]$, e o inverso para o nível da escolaridade, com prevalência mais elevada nos indivíduos analfabetos $/ 1^{\circ}$ ciclo, e menor prevalência nos indivíduos com o ensino superior $[55,1 \%$ vs $16,5 \%$, RP $=3,34$ (IC 95\%, 2,80 - 3,99), $p<0,001]$.

$\mathrm{Na}$ população total não se verificaram diferenças estatisticamente significativas entre regiões de saúde ou tipologia de área urbana. Relativamente ao estado civil, os solteiros apresentaram menor prevalência de SM que os viúvos [15,1\% vs 52,3\%; RP = 3,46 (IC 95\%, 2,79 - 4,30), $p<0,001]$. A proporção de indivíduos com SM foi menor entre aqueles com trabalho remunerado comparativamente às pessoas sem atividade profissional $[24,5 \%$ vs $54,2 \%$; RP $=2,22(\mathrm{IC} 95 \%, 1,03-2,54), p<0,001]$. Os indivíduos que 
declararam não conseguir suportar uma despesa inesperada apresentaram valores superiores de prevalência $[37,4 \%$ vs $31,3 \%, \mathrm{RP}=1,19$ (IC 95\%, 1,04 - 1,37), $p=0,016]$.

$\mathrm{Na}$ estratificação por sexo mantiveram-se as tendências verificadas na população total, a prevalência da SM foi mais elevada nos indivíduos mais idosos, com menor escolaridade, viúvos e sem atividade profissional. Apenas nas mulheres se observou uma maior prevalência entre aquelas incapazes de fazer face a uma despesa inesperada $[36,1 \%$ vs $28,0 \%$; RP $=1,29$ (IC 95\%, 1,10 - 1,51), $p=$ $0,003]$.

\section{Modelo de regressão multivariável}

A análise das razões de prevalências ajustadas (aRP) na Tabela 4 permite verificar que, tanto na população total como em cada um dos sexos, a prevalência foi superior nos indivíduos com mais idade e menor grau de escolaridade.
Em comparação com o grupo etário 25 - 44 anos, a maior aRP observou-se no grupo etário 65 - 74 anos [4,15 (IC $95 \%, 3,51-4,91), p<0,001]$, sendo esta diferença mais pronunciada nas mulheres $[6,94$ (IC 95\%, 5,23 - 9,22), $p$ $<0,001]$ que nos homens [2,71 (IC 95\%, 2,31 - 2,19), $p<$ $0,001]$. Manteve-se também a significância estatística na associação entre SM e nível de escolaridade mais baixo, com uma maior aRP entre os indivíduos analfabetos $/ 1^{\circ} \mathrm{ci}-$ clo [1,76 (IC 95\%, 1,46 - 2,11), $p<0,001]$, comparativamente àqueles com o ensino superior. Quanto ao estado civil, tendo como referência os solteiros, a aRP foi superior nas mulheres viúvas [1,31 (IC 95\%, 1,06 - 1,62), $p=0,018] \mathrm{e}$ nos homens casados ou em união de facto [1,76 (IC 95\%, $1,24-2,50), p=0,004]$. Não se verificaram diferenças estatisticamente significativas entre as categorias das variáveis 'sexo', 'região de saúde', 'tipologia de área urbana', 'trabaIho' e 'risco de pobreza'.

Tabela 2 - Distribuição das caraterísticas analíticas, tensão arterial e medições antropométricas da amostra de indivíduos entre 25 e 74 anos de Portugal ( $n=4797$ ), estratificadas por sexo. (2015)

\begin{tabular}{|c|c|c|c|c|}
\hline Variável & $\begin{array}{c}\text { Total } \\
\mathrm{n}=4797\end{array}$ & $\begin{array}{c}\text { Feminino } \\
n=2571\end{array}$ & $\begin{array}{c}\text { Masculino } \\
n=2226\end{array}$ & $p^{*}$ \\
\hline \multicolumn{5}{|l|}{ Perímetro da cintura (cm) } \\
\hline Média & 92,8 & 89,3 & 96,9 & \multirow{5}{*}{$<0,001$} \\
\hline Desvio padrão & 13,4 & 13,1 & 12,6 & \\
\hline Mediana & 92,1 & 88,6 & 96,1 & \\
\hline Mín - Máx & $57,5-194,0$ & $57,5-148,0$ & $66,0-194,0$ & \\
\hline Aumentado $(q \geq 80 \mathrm{~cm} ; \hat{o} \geq 94 \mathrm{~cm})(\%)$ & 64,5 & 74,4 & 57,4 & \\
\hline \multicolumn{5}{|l|}{ Tensão arterial sistólica (mmHg) } \\
\hline Média & 125,8 & 121,4 & 130,9 & \multirow{4}{*}{$<0,001$} \\
\hline Desvio padrão & 16,7 & 16,5 & 15,4 & \\
\hline Mediana & 124,0 & 119,0 & 129,0 & \\
\hline Mín - Máx & $87,5-224,0$ & $87,5-216,0$ & $88,5-224,0$ & \\
\hline \multicolumn{5}{|l|}{ Tensão arterial diastólica (mmHg) } \\
\hline Média & 74,2 & 72,4 & 76,3 & \multirow{5}{*}{$<0,001$} \\
\hline Desvio padrão & 9,9 & 9,4 & 10,1 & \\
\hline Mediana & 73,5 & 71,5 & 75,5 & \\
\hline Mín - Máx & $43,5-128,0$ & $43,5-119,0$ & $47,0-128,0$ & \\
\hline Aumentada ( $\geq 130 / 85 \mathrm{mmHg}$ ) (\%) & 49,2 & 41,4 & 58,2 & \\
\hline \multicolumn{5}{|l|}{ Trigliceridemia (mg/dL) } \\
\hline Média & 150,7 & 131,9 & 172,4 & \multirow{5}{*}{$<0,001$} \\
\hline Desvio padrão & 99,9 & 76,5 & 117,7 & \\
\hline Mediana & 126,0 & 114,0 & 144 & \\
\hline Mín - Máx & $17,0-1680,0$ & $25,0-885,0$ & $17,0-1680,0$ & \\
\hline Aumentada ( $\geq 175 \mathrm{mg} / \mathrm{dL})(\%)$ & 28,5 & 20,9 & 37,2 & \\
\hline \multicolumn{5}{|l|}{ c-HDL no sangue (mg/dL) } \\
\hline Média & 54,4 & 58,7 & 49,25 & \multirow{5}{*}{$<0,001$} \\
\hline Desvio padrão & 14,5 & 13,9 & 13,6 & \\
\hline Mediana & 53,0 & 57,0 & 47,0 & \\
\hline Mín - Máx & $17,0-171,0$ & $23,0-130,0$ & $17,0-171,0$ & \\
\hline 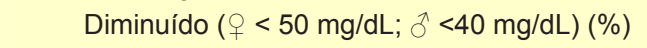 & 24,2 & 26,7 & 21,2 & \\
\hline \multicolumn{5}{|l|}{ HbA1c no sangue (\%) } \\
\hline Média & 5,5 & 5,4 & 5,6 & \multirow{5}{*}{$<0,001$} \\
\hline Desvio padrão & 0,8 & 0,7 & 0,9 & \\
\hline Mediana & 5,4 & 5,3 & 5,4 & \\
\hline Mín - Máx & $3,7-14,1$ & $4,0-12,8$ & $3,7-14,1$ & \\
\hline Aumentada $(\mathrm{HbA} 1 \mathrm{c} \geq 5,7 \%)(\%)$ & 27,4 & 26,0 & 29,1 & \\
\hline
\end{tabular}

C-HDL: colesterol de lipoproteína de alta densidade; HbA1c: hemoglobina glicada A1c; IC 95\%: intervalo de confiança a 95\%

* Comparação de médias entre sexo feminino e masculino 
Tabela 3 - Prevalência de síndrome metabólica e respetivos intervalos de confiança a 95\% (IC 95\%), por caraterísticas demográficas e socioeconómicas, estratificada por sexo e ponderada para a população portuguesa entre 25 e 74 anos. (2015)

\begin{tabular}{|c|c|c|c|c|c|c|c|c|c|}
\hline \multirow{2}{*}{ Variável } & \multicolumn{3}{|c|}{ Total } & \multicolumn{3}{|c|}{ Feminino } & \multicolumn{3}{|c|}{ Masculino } \\
\hline & SM (\%) & IC $95 \%$ & $p$ & SM $(\%)$ & IC $95 \%$ & $p$ & SM $(\%)$ & IC $95 \%$ & $p$ \\
\hline Total & 33,4 & $31,7-35,1$ & - & 31,3 & $28,5-34,2$ & - & 35,6 & $31,9-39,2$ & - \\
\hline \multicolumn{10}{|l|}{ Sexo } \\
\hline Feminino & 31,3 & $28,5-34,2$ & 0,140 & - & - & - & - & - & - \\
\hline Masculino & 35,6 & $31,9-39,2$ & & - & - & - & - & - & - \\
\hline \multicolumn{10}{|l|}{ Grupo etário } \\
\hline $25-44$ & 14,5 & $12,3-16,6$ & $<0,001$ & 9,3 & $6,7-11,9$ & $<0,001$ & 19,9 & $16,2-23,7$ & $<0,001$ \\
\hline $45-54$ & 32,7 & $28,5-36,9$ & & 28,1 & $21,6-34,5$ & & 37,6 & $29,2-49,0$ & \\
\hline $55-64$ & 53,8 & $48,3-59,2$ & & 54,2 & $48,5-60,0$ & & 53,2 & $45,9-60,6$ & \\
\hline $65-74$ & 59,8 & $55,3-64,2$ & & 64,5 & $56,3-72,6$ & & 54,1 & $48,3-59,9$ & \\
\hline \multicolumn{10}{|l|}{ Região } \\
\hline Norte & 34,2 & $35,5-36,0$ & 0,207 & 34,0 & $23,4-38,6$ & 0,263 & 34,5 & $27,8-41,3$ & 0,578 \\
\hline Centro & 37,4 & $30,6-44,1$ & & 34,1 & $26,5-41,7$ & & 40,9 & $33,5-48,4$ & \\
\hline Lisboa e Vale do Tejo & 31,1 & $28,4-33,8$ & & 28,3 & $22,8-33,7$ & & 34,1 & $27,1-41,1$ & \\
\hline Alentejo & 34,3 & $27,6-41,0$ & & 30,0 & $23,7-36,3$ & & 38,6 & $27,6-49,5$ & \\
\hline Algarve & 31,1 & $25,9-36,2$ & & 25,9 & $18,5-33,3$ & & 36,5 & $31,0-42,0$ & \\
\hline Região Autónoma da Madeira & 28,6 & $23,3-33,9$ & & 28,1 & $22,7-33,4$ & & 29,3 & $22,9-35,6$ & \\
\hline Região Autónoma dos Açores & 32,2 & $27,8-36,7$ & & 29,6 & $25,8-33,4$ & & 34,9 & $25,9-44,0$ & \\
\hline \multicolumn{10}{|l|}{ Tipologia área urbana } \\
\hline Rural & 36,6 & $31,4-41,7$ & 0,068 & 36,3 & $27,3-45,4$ & 0,099 & 36,8 & $25,9-47,8$ & 0,731 \\
\hline Urbana & 32,2 & $30,7-33,6$ & & 29,6 & $27,2-31,9$ & & 35,1 & $32,0-38,3$ & \\
\hline \multicolumn{10}{|l|}{ Estado civil } \\
\hline Solteiro & 15,1 & $12,5-17,7$ & $<0,001$ & 13,6 & $8,8-16,5$ & $<0,001$ & 16,4 & $10,2-22,6$ & $<0,001$ \\
\hline Casado ou União de facto & 38,1 & $36,3-40,0$ & & 34,7 & $31,4-38,0$ & & 41,8 & $37,4-46,3$ & \\
\hline Divorciado & 26,9 & $20,5-33,3$ & & 23,1 & $12,5-33,6$ & & 31,1 & $24,0-38,2$ & \\
\hline Viúvo & 52,3 & $41,1-63-6$ & & 52,7 & $41,9-63,5$ & & 50,8 & $25,3-76,3$ & \\
\hline \multicolumn{10}{|l|}{ Escolaridade } \\
\hline Analfabeto $/ 1^{\circ}$ ciclo & 55,1 & $51,9-58,3$ & $<0,001$ & 58,4 & $52,7-64,2$ & $<0,001$ & 51,2 & $46,6-55,9$ & $<0,001$ \\
\hline $2^{\circ}$ ciclo $/ 3^{\circ}$ ciclo & 30,2 & $26,6-33,9$ & & 27,6 & $20,9-34,2$ & & 32,4 & $25,8-39,1$ & \\
\hline Secundário & 25,8 & $22,2-29,3$ & & 18,5 & $13,8-23,2$ & & 33,3 & $28,1-38,6$ & \\
\hline Ensino superior & 16,5 & $13,6-19,4$ & & 14,0 & $9,1-19,0$ & & 20,4 & $13,2-27,7$ & \\
\hline \multicolumn{10}{|l|}{ Trabalho } \\
\hline Trabalho remunerado & 24,5 & $22,3-26,6$ & $<0,001$ & 18,5 & $15,1-21,9$ & $<0,001$ & 30,3 & $25,4-35,1$ & $<0,001$ \\
\hline Desempregado & 33,9 & $28,4-39,4$ & & 29,2 & $20,8-37,6$ & & 39,5 & $32,6-46,3$ & \\
\hline Outra sem atividade profissional & 54,2 & $50,1-58,3$ & & 57,9 & $52,4-63,4$ & & 49,0 & $43,4-54,7$ & \\
\hline \multicolumn{10}{|l|}{ Risco pobreza } \\
\hline Não suporta despesa inesperada & 37,4 & $33,4-41,4$ & 0,018 & 36,1 & $30,7-41,6$ & 0,005 & 39,2 & $32,9-45,5$ & 0,237 \\
\hline Suporta despesa inesperada & 31,3 & $29,2-33,4$ & & 28,0 & $25,7-30,3$ & & 34,4 & $29,7-39,1$ & \\
\hline
\end{tabular}

IC 95\%: intervalo de confiança a 95\%; $p$ : valor-p; SM: síndrome metabólica

\section{DISCUSSÃO}

A prevalência da SM estimada no presente estudo $(33,4 \%)$ situou-se entre valores obtidos em outros estudos. O estudo VALSIM $^{9}$ encontrou uma menor prevalência $(27,5 \%)$, que pode estar relacionada com a participação exclusiva de utentes frequentadores dos CSP e com análises clínicas nos 12 meses anteriores, podendo existir um viés de seleção para indivíduos com maior atenção ao seu estado de saúde e com melhor acesso a cuidados. Além disso, nesse estudo foram utilizados pontos de corte superiores nas componentes do perímetro abdominal e glicemia em jejum. O estudo PORMETS ${ }^{8}$ estimou uma maior prevalência de SM segundo a definição harmonizada $(43,1 \%)$, que parece dever-se maioritariamente à inclusão de 


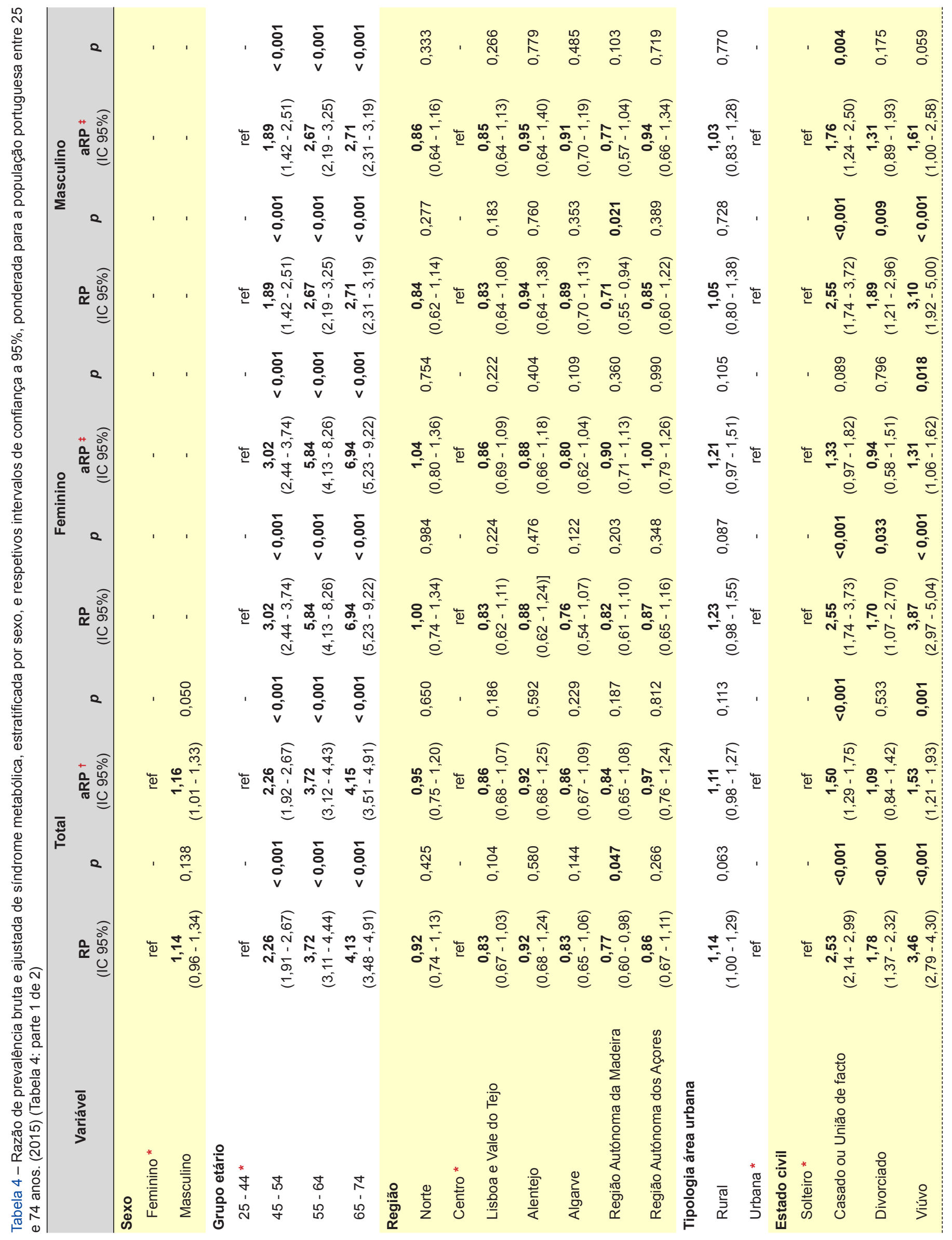


participantes com idade superior aos 74 anos e nos quais a prevalência de SM é mais alta. A nível internacional, outros estudos estimaram prevalências semelhantes em Espanha ${ }^{3}$ (31\%) e Estado Unidos da América ${ }^{13}$ (34\%).

Entre os indivíduos com SM, as componentes mais presentes foram o perímetro da cintura aumentado e HTA, semelhante ao verificado em estudos internacionais realizados pelo consórcio Metabolic Syndrome and Arteries Research (MARE) ${ }^{7}$ e projeto Monica Risk, Genetics, Archiving and Monograph (MORGAM). ${ }^{27}$ No presente estudo, a frequência da componente de perímetro da cintura aumentado foi mais elevada, possivelmente pela utilização de um ponto de corte mais baixo, decorrente de várias organizações considerarem existir aumento do risco de doenças cardiovasculares e diabetes tipo 2 para valores iguais ou superiores a $94 \mathrm{~cm}$ nos homens e iguais ou superiores a $80 \mathrm{~cm}$ nas mulheres. ${ }^{2}$

$\mathrm{Na}$ análise bivariável, observaram-se diferenças com significado estatístico na prevalência de SM entre as categorias das variáveis 'grupo etário', 'estado civil', 'escolaridade' e 'condição perante o trabalho', tanto na população total como em cada um dos sexos. As diferenças entre sexos verificaram-se no 'risco de pobreza' - significativo apenas para a população total e mulheres. As diferenças entre sexos verificaram-se no 'risco de pobreza' - significativo apenas para a população total e mulheres.

Após ajustamento na análise multivariável, apenas se observaram diferenças para as variáveis 'grupo etário', 'estado civil' e 'escolaridade'. Não se verificou uma diferença na prevalência de SM entre sexos. O estudo do consórcio MARE ${ }^{7}$ observou uma diferença entre sexos muito variável nos países analisados, com uma prevalência global da SM ligeiramente superior no sexo feminino $(24,6 \%$ vs $23,9 \%, p<0,001)$. Os estudos VALSIM e PORMETS verificaram uma maior prevalência da SM nas mulheres, podendo esta diferença dever-se, novamente, ao facto do presente estudo não incluir indivíduos acima dos 74 anos. Os estudos VALSIM e PORMETS encontraram uma prevalência mais elevada nas mulheres apenas nos grupos etários acima dos 49 anos, e esta diferença acentuava-se com 0 aumento da idade ${ }^{9,28}$ Esta maior prevalência da SM nas mulheres apenas nos grupos etários acima dos 49 anos pode dever-se ao aumento acentuado da pressão arterial após a

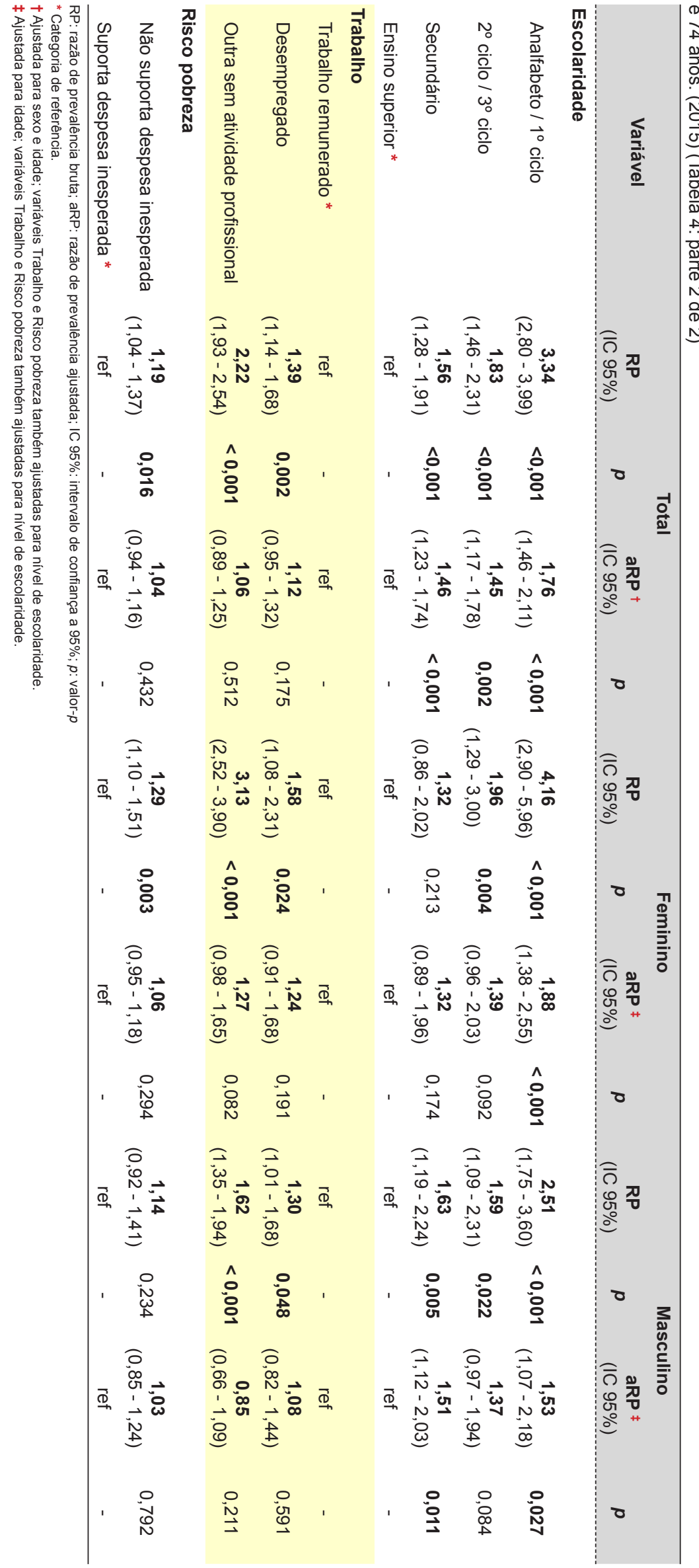

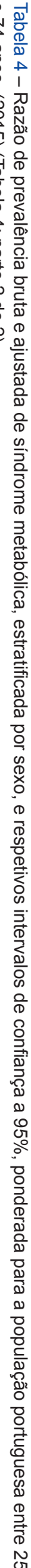


menopausa, que inicia uma diminuição mais rápida da função endotelial nas mulheres. ${ }^{27}$ Isto significa que a SM atinge uma maior proporção de indivíduos do sexo masculino precocemente, expondo-os prolongadamente a estes fatores de risco. Desta forma, será importante ter em consideração este grupo populacional e intervir precocemente para minimizar potenciais consequências a nível metabólico e cardiovascular.

Se tivermos em consideração a variável 'grupo etário', a prevalência da SM é progressivamente maior à medida que aumenta a idade dos indivíduos. Esta associação é transversal a todos os estudos e deve-se em grande parte à acumulação de problemas de saúde relacionados com o envelhecimento, entre eles várias das componentes da SM, como a HTA ou a hiperglicemia.

A presença de SM mostrou-se, nos homens, estar associada ao estado civil casado/unido de facto, e nas mulheres, às viúvas. Relativamente à saúde em geral, a literatura parece indicar que os indivíduos casados têm melhores resultados em saúde comparativamente aos não casados (inclui nunca casados, divorciados e viúvos). ${ }^{29}$ No que respeita especificamente à relação entre estado civil e SM os dados são escassos e, tanto o estudo PORMETS ${ }^{8}$ como outro estudo que utilizou uma amostra de habitantes do Porto, ${ }^{30}$ não encontraram diferenças entre casados e não casados relativamente à prevalência de SM. Além do estado civil, poderão estar envolvidos fatores culturais, psicológicos ou da qualidade do casamento, ${ }^{31}$ que possibilitem a interpretação desta diferença entre sexos. As questões relacionadas com a predominância da síndrome metabólica entre sexos e a sua evolução ao longo do ciclo de vida, bem como os fatores envolvidos nas diferenças encontradas entre os distintos estados civis, merecem estudos mais direcionados e aprofundados para uma melhor compreensão destas temáticas.

No presente estudo foi possível verificar que um baixo nível de escolaridade está associado a uma maior prevalência de SM. Este efeito foi mais evidente nos indivíduos analfabetos $/ 1^{\circ}$ ciclo, sendo mais pronunciado nas mulheres que nos homens. A relação entre educação e saúde é bem conhecida, tendo sido identificada em vários estudos internacionais sobre SM, ${ }^{12-14}$ estando mediada por fatores o como rendimento, acesso a recursos, competências sociais e psicológicas, e literacia. ${ }^{32,33}$

\section{Limitações e potenciais vieses}

Dada a natureza transversal deste estudo, é possível a existência de viés de causalidade reversa, uma vez que não permite estabelecer uma relação temporal entre as associações encontradas.

A possibilidade de viés de seleção foi minimizada pelo desenho da amostra do INSEF que incluiu seleção aleatória dos participantes e estratificação por região e tipologia de área urbana, fortalecendo a sua representatividade. Apesar da taxa de participação (43,9\%) ter sido acima do valor esperado e para o qual o INSEF foi planeado, ${ }^{16}$ podem existir diferenças entre os indivíduos que aceitaram participar e aqueles que não o fizeram. Uma avaliação preliminar não encontrou diferenças significativas entre participantes e não participantes para a maioria das variáveis analisadas. ${ }^{10}$

Um potencial viés de informação prende-se com o facto da definição harmonizada de SM referir que o sangue colhido para análises clínicas deve ser obtido em jejum, mas a participação no INSEF ter ocorrido sem esta obrigatoriedade. Comparando a proporção de indivíduos da amostra que cumprem os critérios das diferentes componentes da SM no presente estudo e nos estudos PORMETS ${ }^{8}$ e VALSIM, ${ }^{9}$ é possível verificar que as maiores diferenças se verificam nas componentes não afetadas pela existência ou ausência de jejum (perímetro da cintura, tensão arterial e colesterol HDL). Na avaliação da trigliceridemia, a proporção é bastante semelhante entre os três estudos $(27,7 \%$ vs $29,4 \%$ vs $30,2 \%$ ), e na componente da glicemia/HbA1c existe maior proximidade entre os valores obtidos no presente estudo e no estudo PORMETS $(27,7 \%$ vs $29,4 \%)$ que no estudo VALSIM (19,0\%). De referir que no estudo VALSIM o ponto de corte para a componente glicemia foi superior (> 110 $\mathrm{mg} / \mathrm{dL}$ ) ao da definição harmonizada (> $100 \mathrm{mg} / \mathrm{dL}$ ), o que pode justificar a menor proporção observada.

No que refere à avaliação da trigliceridemia sem jejum garantido, esta situação já se verificou noutros estudos sobre SM em que os autores referem pontos de corte alternativos entre os $200 \mathrm{mg} / \mathrm{dL}^{34}$ e os $175 \mathrm{mg} / \mathrm{dl}^{20,35,36}$ ou mesmo a utilização do habitual ponto de corte de $150 \mathrm{mg} / \mathrm{dL} .{ }^{37}$ Neste estudo optou-se por substituir o ponto de corte da definição harmonizada (150 mg/dL) por um ponto de corte superior (175 mg/dL), para minimizar erros de classificação.

A glicemia não foi medida no INSEF, tendo sido analisada a $\mathrm{HbA} 1 \mathrm{c}$ como medida indicadora da glicemia média nas últimas oito a 12 semanas. Apesar desta limitação, a evidência atual não sugere excessivos erros de classificação nesta alteração à definição de SM. Assim, neste estudo, a componente da glicemia em jejum $\geq 100 \mathrm{mg} / \mathrm{dL}$ foi substituída pela $\mathrm{HbA} 1 \mathrm{c} \geq 5,7 \%$. Este ponto de corte deriva da definição de pré-diabetes da American Diabetes Association $^{38}$ e outros estudos ${ }^{39,40}$ que, em termos de risco de desenvolver diabetes no futuro, consideram equivalentes a glicemia em jejum $\geq 100 \mathrm{mg} / \mathrm{dL}$ e a $\mathrm{HbA} 1 \mathrm{c} \geq 5,7 \%$. Outros estudos mostraram uma concordância superior a $90 \%$ na classificação dos indivíduos como tendo ou não a SM segundo a definição harmonizada e utilizando a glicemia em jejum $\geq 100 \mathrm{mg} / \mathrm{dL}$ ou a $\mathrm{HbA} 1 \mathrm{c} \geq 5,7 \% .{ }^{41,42}$

A ausência de informação sobre a toma de medicação para hipertrigliceridemia e c-HDL diminuído é também uma limitação deste estudo, podendo originar erros de classificação nestas componentes da SM.

Neste estudo não foram avaliados determinantes de saúde com relevo para a temática da síndrome metabólica como a dieta, atividade física e hábitos tabágicos. Outros parâmetros analíticos com possível relação com a SM, deviam também ter sido levados em conta, nomeadamente, o colesterol de lipoproteínas de baixa densidade e de lipoproteínas de muito baixa densidade, ${ }^{43}$ a uricemia, ${ }^{44}$ a creatininemia ${ }^{45}$ e os níveis de sódio ${ }^{46}$ e potássio. ${ }^{47} \mathrm{~A}$ análise 
destas relações constitui também uma importante área para futuros estudos.

\section{CONCLUSÃO}

A SM afeta uma grande proporção da população portuguesa, constituindo um problema de saúde pública pela sua associação a doenças cardiovasculares e diabetes tipo 2. Este estudo permitiu identificar relações da SM com o sexo, grupo etário, nível de escolaridade e estado civil dos indivíduos. Em especial, o facto da SM ser mais frequente nos indivíduos com menor escolaridade sugere que a educação, para além do impacto que tem no desenvolvimento pessoal e nas relações sociais, pode potenciar a adoção de um estilo de vida mais saudável com repercussões a nível da saúde. Por outro lado, as associações encontradas reforçam a importância de uma abordagem holística que também tenha em conta outros determinantes de saúde e parâmetros analíticos associados à síndrome metabólica e que não foram avaliados neste estudo.

\section{CONTRIBUTO DOS AUTORES}

RA: Concepção do desenho do estudo; análise e interpretação dos dados; redação do artigo.

AJS: Contribuição para a análise e interpretação dos dados; revisão crítica do artigo.

IK: Contribuição para a conceção do estudo; análise e interpretação dos dados; revisão do artigo.

BN: Contribuição para a conceção do estudo; análise e interpretação dos dados.

\section{REFERÊNCIAS}

1. Pereira S, Pereira D. Síndrome metabólico e actividade física. Acta Med Port. 2011;24:785-90.

2. Alberti K, Eckel RH, Grundy SM, Zimmet PZ, Cleeman JI, Donato KA, et al. Harmonizing the metabolic syndrome. Circulation. 2009;120:1640-5.

3. Fernández-Bergés D, Cabrera de León A, Sanz H, Elosua R, Guembe MJ, Alzamora M, et al. Síndrome metabólico en España: prevalencia y riesgo coronario asociado a la definición armonizada y a la propuesta por la OMS. Estudio DARIOS. Rev Española Cardiol. 2012;65:241-8.

4. Mozumdar A, Liguori G. Persistent increase of prevalence of metabolic syndrome among U.S. adults: NHANES III to NHANES 1999-2006. Diabetes Care. 2011;34:216-9.

5. International Diabetes Federation. The IDF consensus worldwide definition of the metabolic syndrome. Brussels: IDF; 2006.

6. Saklayen MG. The global epidemic of the metabolic syndrome. Curr Hypertens Rep. 2018;20:12.

7. Scuteri A, Laurent S, Cucca F, Cockcroft J, Cunha PG, Mañas LR, et al. Metabolic syndrome across Europe: different clusters of risk factors. Eur J Prev Cardiol. 2015;22:486-91.

8. Raposo L, Severo M, Barros H, Santos AC. The prevalence of the metabolic syndrome in Portugal: the PORMETS study. BMC Public Health. 2017;17:555.

9. Fiuza M, Cortez-Dias N, Martins S, Belo A, VALSIM study investigators. Metabolic syndrome in Portugal: prevalence and implications for cardiovascular risk--results from the VALSIM Study. Rev Port Cardiol. 2008;27:1495-529.

10. Gaio V, Antunes L, Namorado S, Barreto M, Gil A, Kyslaya I, et al. Prevalence of overweight and obesity in Portugal: results from the first portuguese health examination survey (INSEF 2015). Obes Res Clin Pract. 2018;12:40-50.

11. Blanquet $M$, Legrand $A$, Pélissier $A$, Mourgues $C$. Socio-economics status and metabolic syndrome: a meta-analysis. Diabetes Metab Syndr Clin Res Rev. 2019;13:1805-12.

12. Silventoinen K, Pankow J, Jousilahti P, Hu G, Tuomilehto J. Educational
ACF: Interpretação dos dados; revisão crítica do artigo; aprovação da versão a publicar.

\section{PROTEÇÃO DE PESSOAS E ANIMAIS}

Os autores declaram que os procedimentos seguidos estavam de acordo com os regulamentos estabelecidos pelos responsáveis da Comissão de Investigação Clínica e Ética e de acordo com a Declaração de Helsínquia da Associação Médica Mundial atualizada em 2013.

\section{CONFIDENCIALIDADE DOS DADOS}

Os autores declaram ter seguido os protocolos do seu centro de trabalho acerca da publicação de dados.

\section{CONFLITOS DE INTERESSE}

Os autores declaram não ter conflitos de interesse.

\section{FONTES DE FINANCIAMENTO}

O $1^{\circ}$ Inquérito Nacional de Saúde com Exame Físico (INSEF 2015), parte integrante do projeto pré-definido do Programa Iniciativas em Saúde Pública "Improvement of epidemiological health information to support public health decision and management in Portugal. Towards reduced inequalities, improved health, and bilateral cooperation", beneficia de um apoio financeiro de cerca de $1500000 €$ concedido pelo Mecanismo Financeiro do Espaço Económico Europeu 2009-2014 através das EEA Grants e pelo Governo de Portugal.

inequalities in the metabolic syndrome and coronary heart disease among middle-aged men and women. Int J Epidemiol. 2005;34:327-34.

13. Ford ES, Li C, Zhao G. Prevalence and correlates of metabolic syndrome based on a harmonious definition among adults in the US. J Diabetes. 2010;2:180-93.

14. Vernay M, Salanave B, De Peretti C, Druet C, Malon A, Deschamps $\mathrm{V}$, et al. Metabolic syndrome and socioeconomic status in France: The French Nutrition and Health Survey (ENNS, 2006-2007). Int J Public Health. 2013;58:855-64.

15. Iguacel I, Börnhorst C, Michels N, Breidenassel C, Dallongeville J, González-Gross M, et al. Socioeconomically disadvantaged groups and metabolic syndrome in european adolescents: The HELENA study. J Adolesc Health. 2021;68:146-54.

16. Santos A, Gil A, Kislaya I, Antunes L, Barreto M, Namorado S, et al. $1^{\circ}$ Inquérito nacional de saúde com exame físico (INSEF 2015): Relatório metodológico. (Instituto Nacional de Saúde Doutor Ricardo Jorge (INSA). Lisboa: Instituto Nacional de Saúde Doutor Ricardo Jorge; 2016.

17. Tolenen H. EHES Manual: Part B. Fieldwork procedures. National Institute for Health and Welfare. Published 2016. [consultado $2020 \mathrm{dez}$ 11]. Disponível em: https://www.julkari.fi/handle/10024/131503.

18. Nunes B, Barreto M, Gil AP, Kislaya I, Namorado S, Antunes L, et al. The first Portuguese National Health Examination Survey (2015): design, planning and implementation. J Public Health. 2019;41:511-7.

19. Eurostat. Income and living conditions. Comissão Europeia. [consultado 2019 jun 3]. Disponível em: https://ec.europa.eu/eurostat/cache/ metadata/en/ilc_esms.htm.

20. Nordestgaard BG, Langsted A, Mora S, Kolovou G, Baum H, Bruckert $E$, et al. Fasting is not routinely required for determination of a lipid profile: clinical and laboratory implications including flagging at desirable concentration cut-points - a joint consensus statement from the European Atherosclerosis Society and European Federa. Eur Heart J. 2016;37:1944-58 
21. Courtenay WH. Constructions of masculinity and their influence on men's well-being: a theory of gender and health. Soc Sci Med. 2000;50:1385401.

22. White A, McKee M, Richardson N, Visser R de, Madsen SA, Sousa $\mathrm{BC}$ de, et al. Europe's men need their own health strategy. BMJ. 2011;343:d7397.

23. Rao JN, Scott AJ. On simple adjustments to chi-square tests with sample survey data. Ann Stat. 1987;15:385-97.

24. Aguiar $P$, Baltazar N. Odds ratio: reflexão sobre a validade de uma medida de referência em epidemiologia. Acta Med Port. 2013;26:50510

25. R: The R Project for Statistical Computing. [consultado 2020 dez 11] Disponivel em: https://www.r-project.org/.

26. CRAN - Package survey. [consultado 2020 dez 11]. Disponível em: https://cran.r-project.org/web/packages/survey/index.html.

27. Vishram JK, Borglykke A, Andreasen AH, Jeppesen J, Ibsen $H$, Jørgensen $\mathrm{T}$, et al. Impact of age and gender on the prevalence and prognostic importance of the metabolic syndrome and its components in europeans. The Morgam Prospective Cohort Project. Lazzeri C, editor. PLoS One. 2014;9:e107294.

28. Cortez-Dias N, Martins S, Belo A, Fiuza M, Investigadores do estudo VALSIM. Comparison of definitions of metabolic syndrome in relation to risk for coronary artery disease and stroke. Rev Port Cardiol. 2011;30:139-69.

29. Robards J, Evandrou M, Falkingham J, Vlachantoni A. Marital status, health and mortality. Maturitas. 2012;73:295-9.

30. Santos AC, Ebrahim S, Barros H. Gender, socio-economic status and metabolic syndrome in middle-aged and old adults. BMC Public Health. 2008;8:62.

31. Lawrence EM, Rogers RG, Zajacova A, Wadsworth T. Marital happiness, marital status, health, and longevity. J Happiness Stud. 2019;20:153961.

32. Hahn RA, Truman BI. Education improves public health and promotes health equity. Int J Health Serv. 2015;45:657-78.

33. Baker DP, Leon J, Smith Greenaway EG, Collins J, Movit M. The education effect on population health: a reassessment. Popul Dev Rev. 2011;37:307-32

34. Miller M, Stone NJ, Ballantyne C, Bittner V, Criqui MH, Ginsberg HN, et al. Triglycerides and cardiovascular disease. Circulation. 2011;123:2292333.

35. Scartezini M, Ferreira CE, Izar MC, Bertoluci M, Vencio S, Campana
GA, et al. Positioning about the flexibility of fasting for lipid profiling. Arq Bras Cardiol. 2017;108:195-7.

36. White KT, Moorthy MV, Akinkuolie AO, Demler O, Ridker PM Cook NR, et al. Identifying an optimal cutpoint for the diagnosis of hypertriglyceridemia in the nonfasting state. Clin Chem. 2015;61:115663.

37. Sundvall J, Leiviskä J, Laatikainen T, Peltonen M, Salomaa V, Vanhala $M$, et al. The use of fasting vs. non-fasting triglyceride concentration for estimating the prevalence of high LDL-cholesterol and metabolic syndrome in population surveys. BMC Med Res Methodol. 2011;11:63.

38. American Diabetes Association. Standards of medical care in diabetes 2019. Diabetes Care. 2019;42:S17-18.

39. Zhang X, Gregg EW, Williamson DF, Barker LE, Thomas W, Bullard KM, et al. A1C level and future risk of diabetes: a systematic review. Diabetes Care. 2010;33:1665-73.

40. Veeranna V, Ramesh K, Zalawadiya SK, Niraj A, Pradhan J, Jacob $\mathrm{S}$, et al. Glycosylated hemoglobin and prevalent metabolic syndrome in nondiabetic multiethnic U.S. adults. Metab Syndr Relat Disord. 2011:9:361-7.

41. Ong KL, Tso AW, Lam KS, Cherny SS, Sham PC, Cheung BM. Using glycosylated hemoglobin to define the metabolic syndrome in United States adults. Diabetes Care. 2010;33:1856-8.

42. Succurro E, Marini MA, Arturi F, Grembiale A, Fiorentino TV, Andreozzi $F$, et al. Usefulness of hemoglobin $A 1 C$ as a criterion to define the metabolic syndrome in a cohort of italian nondiabetic white subjects. Am J Cardiol. 2011;107:1650-5.

43. Adiels M, Olofsson SO, Taskinen MR, Borén J. Overproduction of very low-density lipoproteins is the hallmark of the dyslipidemia in the metabolic syndrome. Arterioscler Thromb Vasc Biol. 2008;28:1225-36.

44. Kanbay M, Jensen T, Solak Y, Le M, Roncal-Jimenez C, Rivard C, et al. Uric acid in metabolic syndrome: from an innocent bystander to a central player. Eur J Intern Med. 2016;29:3-8.

45. Wang J, Li X, Han X, Yang K, Liu B, Li Y, et al. Serum creatinine levels and risk of metabolic syndrome in a middle-aged and older Chinese population. Clin Chim Acta. 2015;440:177-82.

46. Oh SW, Han KH, Han SY, Koo HS, Kim S, Chin HJ. Association of sodium excretion with metabolic syndrome, insulin resistance, and body fat. Medicine. 2015;94:e1650.

47. Shin D, Joh HK, Kim KH, Park SM. Benefits of potassium intake on metabolic syndrome: the fourth Korean national health and nutrition examination survey (KNHANES IV). Atherosclerosis. 2013;230:80-5. 